\title{
Automated Machine Learning algorithm for Kidney and Kidney tumor segmentation in Computed Tomography Scans
}

\author{
Bharadwaj Kss, Vivek Pawar \\ Endimension Technology Private Limited, Mumbai, India
}

\begin{abstract}
In this report,we have described an automated algorithm for accurate segmentation of kidney and kidney tumor from CT scans. The dataset for this problem was made available online as part of KiTS19 Challenge. Our model uses a 2 stage cascaded Residual Unet architecture. The first network is designed to predict (Kidney + Tumor) regions. The second network predicts segmented tumor regions from the output of first net. As a post processing step, we have designed a statistical metric which calculates the standard deviation of derivatives of centre of mass of predicted masks to filter out false positives. The report contains implementation details along with results on validation set.
\end{abstract}

Keywords: First keyword · Second keyword · Another keyword.

\section{Introduction}

There are more than 400,000 new cases of kidney cancer each year [1], and surgery is its most common treatment [2]. KiTS19 challenge [3] was conducted to accelerate the development of reliable kidney and kidney tumor semantic segmentation methodologies. Ground truth semantic segmentations for arterial phase abdominal CT scans of 210 unique kidney cancer patients were provided as part of the training dataset for the challenge. The submission models are then evaluated on a hidden test dataset of 90 patients.

\section{Pre Processing}

The training dataset for KiTS19 challenge is made available online [4]. The GitHub Repository had two branches, master and interpolated. Master has the data at the spacing at which it was captured and interpolated has the data at the median pixel width $(0.78162497 \mathrm{~mm})$ and slice thickness $(3.0 \mathrm{~mm})$. We downloaded the data from the master branch. Later, we interpolated the data to have a uniform slice thickness of $3 \mathrm{~mm}$. A final pre-processing step was to scale each input feature using min-max normalization. 


\section{Network Architecture}

We used a two stage cascaded Unet Architecture for effectively segmenting both kidney and kidney tumor. The first network is designed to look at the entire CT scan and output (Kidney + Tumor) regions. These output regions are then fed to the second network which has been designed to segment tumor regions from (Kidney + Tumor) regions. For both the stages, the base network is a Unet architecture as illustrated in Figure 1. To be able to effectively train deep networks, we have used residual connections on top of U-Net.The residual connections are as described in Figure 2

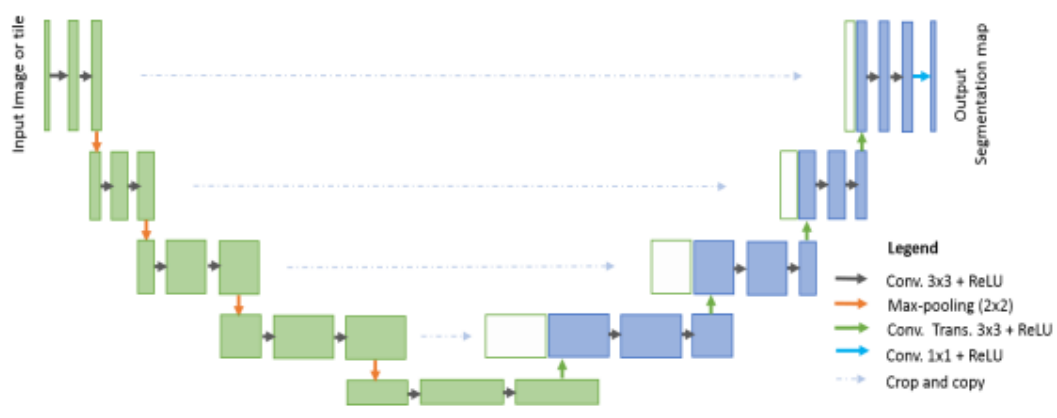

Fig. 1. Base U-Net architecture with convolutional encoding and decoding units that take image as input and produce the segmentation feature maps

\section{Implementation Details}

For the first stage, we used a 2D Residual U-Net. The input feature size to the first net is $(10,5,512,512)$ where batch_size $=10$,input_channel_size $=5$,image_width $=512$ and image_height $=512$.

For the second stage,we used a 3D Residual U-Net. The input feature size to the second network is $(10,1,9,224,224)$ where batch_size $=10$, input_channel $=1$, image_depth $=9$, image_width $=224$, image_height $=224$.

Dice loss function, similar to the one used in KiTS19 evaluation metric is used to train both the nets. Adam optimizer is used with a learning rate of $1 \mathrm{e}-3$.

170 patients are used for training both the networks. 38 patients were kept for model validation. Since the data set is small, we have made use of data augmentation to prevent over-fitting. In both models, we have used flip, mirroring,shift,rotation and elastic augmentations. 


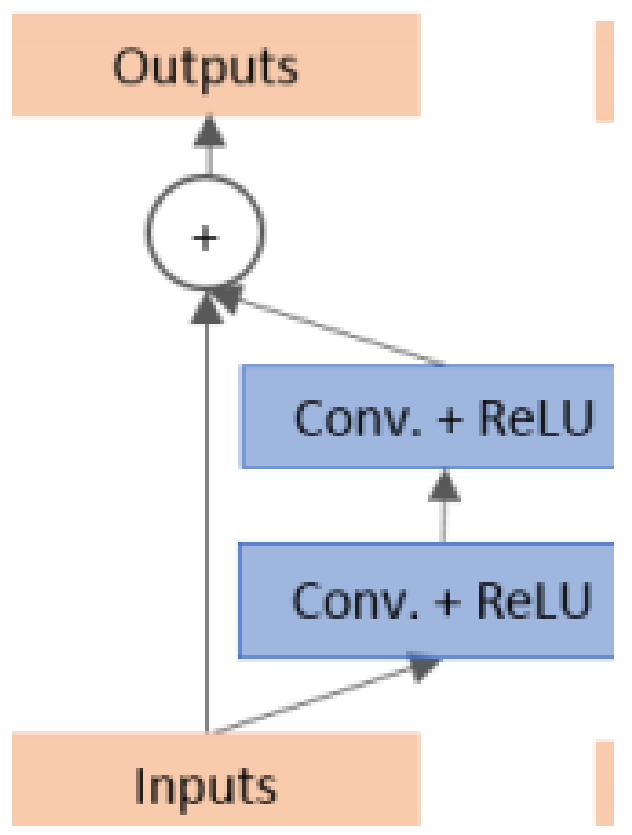

Fig. 2. Residual Convolution Block

\section{Post Processing}

To eliminate false positive regions, we have come up with a novel statistical metric. For each predicted region pair, we calculate standard deviation of the derivatives of centre of mass of prediction masks. Within each patient, we select the pair with the least standard deviation. The prediction probabilities are finally converted into labels by applying thresholds. We used a threshold of 0.5 for (Kidney + Tumor) prediction mask and 0.8 for Tumor prediction mask.

\section{Results}

Our network is very effective at detecting both kidney and tumor in most cases. However, we are generating few false positives which is reflected in the final score. Results on some sample validation cases are shown in Figure 3 and Figure 4 below. 

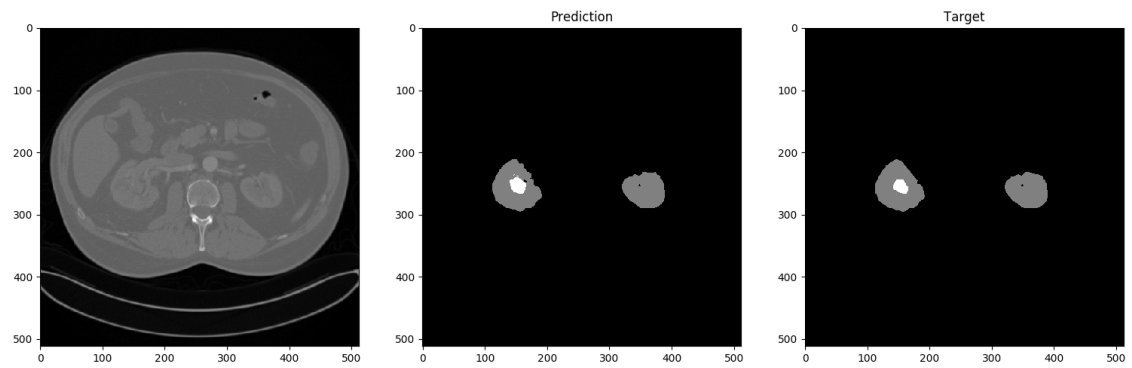

Fig. 3. Experimental outputs from our network. First column shows input slice. Second column shows network predictions and third column is the ground truth
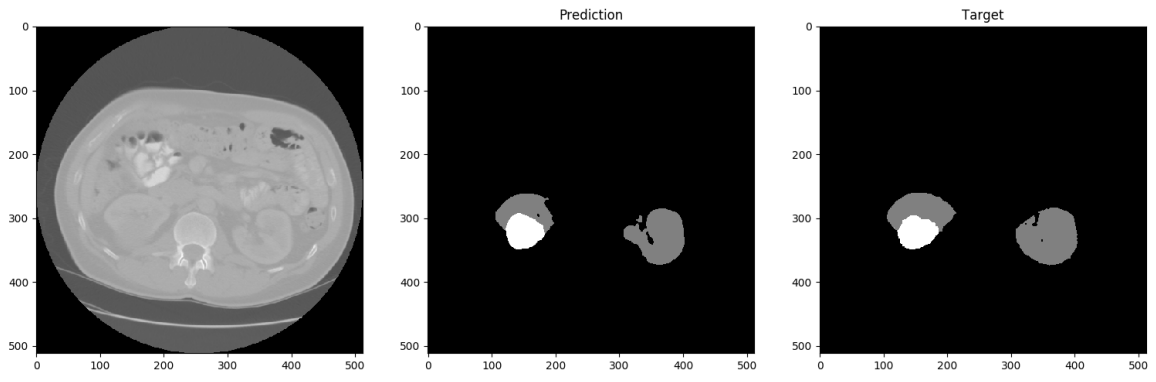

Fig. 4. Experimental outputs from our network. First column shows input slice. Second column shows network predictions and third column is the ground truth

\section{References}

1. "Kidney Cancer Statistics." World Cancer Research Fund, 12 Sept. 2018, www.wcrf.org/dietandcancer/cancer-trends/kidney-cancer-statistics.

2. "Cancer Diagnosis and Treatment Statistics." Stages - Mesothelioma - Cancer Research UK, 26 Oct. 2017, www.cancerresearchuk.org/health-professional/cancerstatistics/diagnosis-and-treatment.

3. "Kits 19 Challenge" https://kits19.grand-challenge.org/home/

4. "KITS19 Github" https://github.com/neheller/kits19 